\title{
Indicadores de violencia relacionados con el ciberbullying en adolescentes del Ecuador
}

\author{
Indicators of Cyberbullying-Related Violence \\ In Teenagers in Ecuador
}

Rodrigo Moreta-Herrera ${ }^{1} \bowtie$, Santiago Poveda-Ríos ${ }^{2}$, Isabel Ramos-Noboa ${ }^{3}$

${ }_{1}$ Pontificia Universidad Católica del Ecuador
${ }^{2}$ Pontificia Universidad Católica del Ecuador
${ }^{3}$ Pontificia Universidad Católica del Ecuador

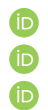

$\triangle A v$. Manuela Sáenz y Remigio Crespo, Ambato, Ecuador. Correo electrónico: rmoreta@pucesa.edu.ec

Recibido: 15 de junio del 2017 Aprobado: 12 de julio del $2018 \quad$ Disponible en línea: 1 de octubre del 2018

Cómo citar este artículo: Moreta-Herrera, R., Poveda-Rios, S. y Ramos-Noboa, M. I. (2018). Indicadores de violencia relacionados con el ciberbullying en adolescentes del Ecuador. Pensando Psicología, 14(24). doi: https://doi.org/10.16925/ pe.v14i24.1895

\section{Resumen}

Objetivo: determinar la presencia de indicadores de ciberbullying en una muestra de adolescentes escolarizados del Ecuador y las prácticas más recurrentes, así como las diferencias existentes por sexo y tipo de institución educativa.

Método: se realizó un estudio descriptivo exploratorio y comparativo con el Cuestionario de Cyberbullying (CBQ) y su complemento de victimización (CBQ-v).

Muestra: participaron 432 estudiantes de bachillerato entre el décimo año de educación básica y el tercer año de bachillerato. El 55,3\% son mujeres y el 44,7\% son hombres, con edades entre 13 y 22 años de edad ( $M=15,3$ años; $D s=1,13)$.

Resultados: la perpetración virtual leve es del 56,9\%, mientras que la de victimización virtual es del $42,8 \%$. La exclusión de grupos, el envío de mensajes humillantes e insultantes y el robo de la identidad de la víctima fueron los indicadores más prevalentes de ciberacoso. No se encontraron diferencias por sexo en cuanto a conductas perpetradoras $(t=-0,18) 0$ de victimización $(t=1,12)$; pero está presente en la prevalencia de conductas perpetradoras $(t=-2,18)$ en estudiantes que provienen de centros educativos privados.

Discusión: los resultados de perpetración y victimización se corresponden parcialmente con los reportados en otros estudios a nivel internacional, pero las variaciones necesitan ser explicadas en consideración de otros factores psicosociales.

Palabras clave: acoso, ciberbullying, perpetración, tecnología, victimización. 


\title{
Indicators of Cyberbullying-Related Violence In Teenagers in Ecuador
}

\begin{abstract} cational institution. $\mathrm{SD}=1.13)$. -2.18) in students who come from private educational institutions.

Keywords: bullying, cyberbullying, perpetration, technology, victimization.

\section{Indicadores de violência relacionados com o ciberbullying em adolescentes do Equador}

Aim: To determine the presence of cyberbullying indicators in a sample of schooled adolescents in Ecuador and the most recurrent practices, as well as the existing differences by sex and type of edu-

Materials and methods: An exploratory, comparative, descriptive study was carried out using the Cyberbullying Questionnaire (СВQ) and its victimization supplement (CBQ-v). Four hundred and thirty-two students in the tenth year of elementary education and third year of high school participated. Fifty-five point three percent are women and $44.7 \%$ are men, with ages between 13 and 22 ( $\mathrm{M}=15.3$ years,

Results: Minor virtual perpetration is $56.9 \%$, while virtual victimization is $42.8 \%$. Group exclusion, sending of humiliating and insulting messages, and theft of the victim's identity were the most prevalent indicators of cyberbullying. No differences were found by sex in terms of perpetrating behavior $(t=-0.18)$ or victimization $(t=1.12)$; but it is present in the prevalence of perpetrating behavior $(t=$

Discussion: The results of perpetration and victimization partially match those reported in other international studies, but the variations need to be explained considering other psychosocial factors.

\section{Resumo}

Objetivo: determinar a presença de indicadores de ciberbullying numa amostra de adolescentes escolarizados do Equador e as práticas mais recorrentes, bem como as diferenças existentes por sexo e tipo de instituiçã̃o educativa.

Método: realizou-se um estudo descritivo exploratório e comparativo com o Questionário de Cyberbullying (СвQ) e seu complemento de vitimização (CBQ-v).

Amostra: participaram 432 estudantes (entre o nono ano do ensino fundamental e o terceiro do médio). $55,3 \%$ são mulheres e 44,7\% são homens, com faixa etária entre 13 e 22 anos de idade $(M=15,3$ anos; $\mathrm{DP}=1,13)$.

Resultados: a perpetração virtual leve é 56,9 \%, enquanto a vitimização virtual é de $42,8 \%$. A exclusão de grupos, 0 envio de mensagens humilhantes e insultantes, e o roubo da identidade da vítima foram os indicadores mais prevalentes de ciberbullying. Não se acharam diferenças significativas por sexo quanto a comportamentos perpetradores $(t=-0,18)$ ou de vitimização $(t=1,12)$; contudo, está presente na prevalência de comportamentos perpetradores $(t=-2,18)$ em estudantes que provêm de centros educativos particulares.

Discussão: os resultados de perpetração e vitimização correspondem parcialmente com os relatados em outros estudos no âmbito internacional, mas as variações precisam ser explicadas em consideração de outros fatores psicossociais.

Palavras-chave: assédio, ciberbullying, perpetração, tecnologia, vitimização. 


\section{Introducción}

Durante las últimas dos décadas, el vertiginoso crecimiento de las nuevas tecnologías y el relativo acceso a ellas genera el desarrollo de nuevas formas de comunicación e interacción entre los individuos, circunscritas en plataformas digitales y dispositivos tecnológicos como computadores, teléfonos móviles y consolas. La masificación de productos electrónicos en los países industrializados, la producción a bajo costo de la tecnología en países en vías de desarrollo y el creciente acceso a internet durante la última década han motivado cambios importantes a nivel educativo, económico y social (Roig, López \& Álvarez, 2017). En el campo educativo, la tecnología trae progreso, pero también facilita el desarrollo del mal uso de la tecnología y la comunicación entre pares, como lo es el ciberbullying o el acoso virtual o cibernético.

El ciberbullying es uno de los fenómenos resultantes de esta revolución digital. Estimaciones generales reportan variaciones en cuanto a la prevalencia de convertirse en una víctima de ciberacoso, aunque se estima entre el 10 y el 40\% (Kowalskiet al., 2014). Una de las regiones con presencia representativa se encuentra en la zona europea, donde en países como Polonia, Suecia, Grecia e Italia se reportan niveles de acoso entre 26 y $28 \%$; y en otros como España, Francia, Portugal y Croacia es aún superior con niveles entre 30 y $42 \%$ de la población internauta (Eurostat, 2015). En la región suramericana, por ejemplo, el $87,8 \%$ de estudiantes chilenos y el $19 \%$ de adolescentes en Argentina entre 12 y 17 años reportaron ser víctimas de acoso por internet o teléfono móvil, mediante mensajes amenazantes y videos (Sevilla, 2011).

Además, en estudios realizados en Argentina, Brasil, Chile, Colombia, México, Perú y Venezuela, se identificó que los comportamientos agresivos se transmitieron a las víctimas potenciales por medio del teléfono celular, las redes sociales y los juegos online aproximadamente en el $12,1 \%$ de los preadolescentes y adolescentes (Del Río et al., 2009). En el Ecuador, aunque los estudios son limitados, ciertos datos reflejan una prevalencia del $11 \%$ aproximadamente entre los adolescentes con diferencias por sexo, con mayor acoso hacia las mujeres. El medio más utilizado son las redes sociales (52\%) y los mensajes por celular (21,7\%) (Mosquera et al., 2016).

El grupo más vulnerable a los efectos del ciberbullying es el de los adolescentes, sin aparentes diferencias por sexo en cuanto a la victimización (Qing, 2006; Gámex-Gaudi, Villa-George y Calvete, 2014).
Y además, esta problemática constituye un factor de riesgo psicosocial entre los adolescentes debido a las dificultades en la interacción social y el contexto académico (Moreta et al., 2017), en parte por el riesgo suicida, la baja autoestima y la depresión que están asociados a la victimización (Chang et al. 2013; Cetin et al., 2012) a los fracasos escolares y a la búsqueda de asistencia médica (Kessel et al., 2012). También, la evidencia muestra la estrecha relación de la perpetración en víctimas de acoso virtual con el rendimiento académico (Yousef y Bellamy, 2015).

La contraparte de la víctima de ciberbullying es la persona acosadora o perpetradora virtual, que comprende al individuo con la práctica de comportamientos intimidatorios ejecutados en función del uso tecnológico y la intención de dañar a los demás (Bauman, Cross y Walker, 2013). Se reporta en el contexto escolar que la edad es relevante: a medida que aumenta, también incrementan la práctica de conductas perpetradoras y la participación pasiva u observacional de otros (Garaigordobil, 2015). La prevalencia fluctúa entre el 20 y el $40 \%$ de la práctica de ciberbullying en algún momento de la vida de los adolescentes (Tokunaga, 2010). Según ciertos estudios, como el de Chile en estudiantes de educación media, el 6,4\% admitió ser un perpetardor o acosador cibernético (Varela et al., 2018); y en Taiwán fue el 5,8\% de estudiantes de secundaria (Chang et al., 2013). En cuanto al sexo, los estudios aún no son concisos y varían según las distintas realidades. En la población mexicana, la práctica de ciberbullying en algún momento de la vida se registra en al menos $42 \%$, con una mayor prevalencia en hombres que en mujeres (Gámex-Gaudi, Villa-George y Calvete, 2014).

\section{Actores, medios y prácticas de ciberbullying}

Para muchas legislaciones, cualquier acto de violencia en el interior de las escuelas similar al bullying es sancionado como un acto punitivo y es juzgado como delito contra la integridad de una o varias personas, pues incluye un comportamiento intencional reiterativo de daño en posición de desventaja (Di Napoli, 2016). Los actores de la violencia escolar están claramente definidos en víctimas, agresores o acosadores y espectadores, cada uno de ellos con características particulares, aunque hay que recalcar que estas varían significativamente cuando el acoso se produce en la convivencia en la escuela o por medio de la tecnología en asuntos competentes a la 
escolaridad (Castillo-Pulido, 2011). Las víctimas pueden definirse como pasivas o activas, con actitudes ansiosas e inseguras, baja autoestima y percepción de fracaso en el primer caso, y comportándose de forma irritante en el segundo; los agresores, por su parte, se muestran impulsivos, son desafiantes con sus compañeros o incluso con figuras de autoridad, presentan necesidad de dominar a otros y disfrutan haciéndolo y terminan por generar intimidación, humillación, aislamiento y exclusión de sus víctimas; y finalmente los espectadores, por su rol en el acoso, son considerados como agresores pasivos (Olweus, 2016).

Los medios por los que se transmite el acoso son aquellos que los adolescentes utilizan con más frecuencia para comunicarse con sus pares (redes sociales, mensajes de texto, llamadas, etc.) y que sirven para el intercambio de información a través de plataformas digitales de descarga popular para el relacionamiento interactivo en la red (Rodríguez-Correa y Arroyo, 2014). Las diversas plataformas digitales prestan tanto ventajas como una amplia gama de desventajas para aquellos involucrados directa o indirectamente con la tecnología; los usuarios han encontrado un mundo infinito de posibilidades para satisfacer sus múltiples necesidades (Castells, 2010).

La tecnología ha modificado sustancialmente la forma de mejorar los sistemas de producción, salud y economía, entre otros; pero concomitante a estos beneficios, se han forjado nuevas formas de acceder a información para realizar fraudes, desfalcos, clonar identidades, propagar virus informáticos, spam o paquetes de información contaminada y más delitos o contravenciones (Donegan, 2012). Las personas abusadoras y acosadoras utilizan esto a su favor, explotan la ingenuidad, curiosidad e irresponsabilidad de los adolescentes para atacarlos con su propia información o con información de interés vinculada al ámbito académico (Shu-Ling, Renmin y Kuo-Pao, 2008).

En las escuelas, uno de los medios más utilizados para el acoso virtual son los teléfonos celulares (Pierskalla y Hollenbach, 2013), debido a sus características de acceso a internet, ya que este dispositivo presta mayores facilidades para hostigar e intimidar a las víctimas, por lo cual el uso irresponsable de estos con fines de causar cualquier tipo de lesión a la dignidad e intimidad de las personas está siendo tratado en la última década como un asunto punitivo con perjuicio legal (Simone, Smith y Blumberg, 2012).
Entre las formas más usuales de acoso o ataque personal se encuentran la difusión de información beligerante vía redes sociales, mensajes de texto, salas de chat, videos, fotografías, correos electrónicos y videollamadas, entre muchas otras (Singhal y Bansal, 2013). La agresión es emitida por medios digitales con videollamadas intimidatorias o vergonzantes; mensajes de texto ofensivos, de calumnia o de ridiculización (Rivers y Noret, 2010); el envío de correos electrónicos con información amenazante o comprometedora; insultos, bloqueos, burlas o engaños en salas de chat (Ybarra y Mitchell, 2004); difusión de videos o fotografías con contenido íntimo personal o de otras personas (Livingstone y Smith, 2014); creación o replicación de memes; propagación de rumores, mentiras y bromas malintencionadas; creación de perfiles falsos en plataformas digitales para engañar, aprovecharse o dañar a otras personas; y muchas variaciones (De Tejada, Muñoz y Rus, 2018). Mientras que las agresiones recibidas por las víctimas comprenden las llamadas silenciosas o de broma y los insultos por mensajes de texto calificadas como leves; y la publicación de fotos o videos íntimos en sitios web y/o escenas de violencia calificadas como severas o graves (Menesini, Nocentini y Calussi, 2011).

\section{Objetivo e hipótesis}

Tras la revisión preliminar del tema, se establecen como objetivos del presente estudio: a) conocer la prevalencia de conductas de perpetración y de victimización cibernética, ya que se estima que esta tiene una considerable presencia; b) conocer las prácticas más recurrentes de perpetración realizadas y de más afectación, pues se desconoce sobre este fenómeno; y c) conocer las posibles diferencias presentes por sexo y por tipo de institución educativa a donde pertenecen los evaluados, ya que se hipotetiza que los hombres muestran mayor componente perpetrador y las mujeres un mayor componente victimizante.

\section{Método}

\section{Diseño}

El presente trabajo se enmarca como un estudio empírico con metodología cuantitativa, de tipo descriptivo, mediante un código arbitrario de observación (muestreo no probabilístico) natural (Montero y 
León, 2007). Se busca describir la presencia de conductas perpetradoras relacionadas con el ciberbullying, así como de los indicadores de victimización. Además, es un análisis bivariado (comparativo), considerando como variables de agrupación el sexo (masculino y femenino) y el tipo de financiación de la institución educativa (pública y privada) en la que cursan sus estudios los participantes.

\section{Participantes}

Se consideraron 432 casos de estudiantes de cinco unidades educativas de la ciudad de Ambato y que formaron parte del proyecto de vinculación con la colectividad, ejecutado por la PUCE-Ambato. La población estimada es de 2000 estudiantes, que comprende a los participantes beneficiarios, por lo que el tamaño de la muestra es representativo.

Entre las características sociodemográficas se observó que $55,3 \%$ son mujeres y $44,7 \%$ son hombres. Las edades de los participantes fueron entre los 13 años y los 22 años $(M=15,25$ años; $D s=1,13)$. Además, los participantes son cursantes de décimo nivel de educación básica (15,5\%), y de primero $(46,1 \%)$, segundo $(28,5 \%)$ y tercero de bachillerato (10\%). Los participantes que proceden de centros educativos privados representan el 20,4\%, mientras que el 79,6\% restante proceden de centros públicos.

El muestreo de los participantes fue de tipo no probabilístico arbitrario con los siguientes criterios de inclusión: a) ser estudiante regular de la institución educativa; b) contar con el consentimiento firmado por los representantes legales o tutores; c) edad comprendida superior a los 13 años; y d) cursar el décimo año de educación básica o algún año de bachillerato.

\section{Instrumentos}

El Cuestionario de Cyberbullying ( $\mathrm{CBQ}$ ) fue diseñado inicialmente por Calvete et al. (2010) en lengua inglesa y cuenta con dos subescalas correlacionadas. El primero es para medir el nivel de perpetración y una subescala complementaria ( $\mathrm{CBQ}-\mathrm{v})$ que mide la victimización ocasionada por el ciberbullying (Estévez et al., 2010).

La versión utilizada corresponde a la versión reducida y adaptada al castellano por los mexicanos Gámex-Gaudi, Villa-George y Calvete (2014). La escala de perpetración cuenta con catorce ítems y la de victimización tiene nueve ítems. Las respuestas a las preguntas fueron formuladas en una escala Likert de cuatros niveles: 0 (nunca), 1 (una o dos veces), 2 (tres o cuatro veces) y 3 (cinco o más veces). Entre la justificación psicométrica de la prueba se puede identificar un buen ajuste al modelo de dos factores correlacionados con $\mathrm{N}=1491$ es $\mathrm{X} 2(220)=293$; $\mathrm{p}<0,001 ; \mathrm{NNFI}=0,98 ; \mathrm{CFI}=0,99 ; \mathrm{RMSEA}=0,03 \mathrm{CI}$ : $95 \%$ $[0,027,0,034]$. La consistencia interna muestra que es de $\alpha=0,81$ para perpetración y de $\alpha=0,59$ para victimización, con una correlación entre los factores de $r=0,71$.

Sobre los resultados preliminares encontrados en la muestra en ecuatorianos, se detalla que la fiabilidad es alta para el comportamiento perpetrador de ciberbullying, con un puntaje de $\alpha=0,849$; mientras que en el caso de los indicadores de victimización por la perpetración la consistencia es de $\alpha=0,783$, equivalente a moderadamente alta. Estudios similares han sido encontrados en población española (GámezGuadix, Gini y Calvete, 2015)

\section{Procedimiento metodológico}

El desarrollo del estudio comprendió la evaluación psicológica diagnóstica, como parte de la gestión del proyecto de vinculación con la colectividad que tuvo la PUCE-Ambato con las instituciones adherentes sobre la prevención del ciberbullying en los colegios. Por eso, contó con las respectivas autorizaciones de las instituciones participantes, junto con la aceptación de los tutores para la implementación del programa y la difusión de los resultados.

La implementación de las evaluaciones psicológicas se hizo en los propios centros de estudio, de manera grupal y en un tiempo aproximado de 10 minutos. Antes se explicaron los objetivos de la evaluación para el desarrollo del proyecto en sí. Después, se realizó una depuración de la información, la sistematización en bases de datos, la gestión informática, la verificación de las hipótesis, los informes de los resultados y el material de difusión que fue supervisado con anterioridad por la Comisión de Publicaciones de la PUCE-Ambato.

\section{Análisis de resultados}

El estudio involucró un análisis de prevalencia e intensidad de los ítems que conforman la escala de perpetración y de victimización para conocer las 
conductas más practicadas. También se realizó un análisis categórico para conocer los distintos niveles de perpetración y victimización existentes, así como la asociación (Chi cuadrado de Pearson) y la correlación (Tau-b de Kendall) existentes entre ambas.

Un tercer análisis comprendió la comparación de las puntuaciones medias por sexo (masculino y femenino) y por tipo de financiamiento de la institución educativa (pública o privada), a fin de conocer si existen diferencias estadísticamente significativas $(\mathrm{p}<, 05)$ entre los componentes del СвQ con la prueba t de Student ( $\mathrm{t}$ ), como lo sugieren Pardo y San Martín (2010) con muestras relativamente grandes, en lo que no consideran necesario el cumplimiento del supuesto de normalidad dado por los criterios del teorema del límite central. Y como análisis complementario, se buscó conocer el tamaño del efecto (Cohen, 1994) a través de la prueba de Hedges ajustada (g(ajust.)). Los análisis estadísticos utilizados en el trabajo fueron realizados con el software spss en la versión 21 (Івм Corp., 2012).

\section{Resultados}

\section{Análisis descriptivo}

\section{Análisis sobre conductas de perpetración}

El análisis muestra que las conductas perpetradoras con más prevalencia (igual o mayor al 10\% en el nivel leve) son: a) la exclusión de alguien de un grupo en línea; b) el envío de mensajes amenazantes o insultantes; c) el envío de mensajes amenazantes o insultantes por correo electrónico; d) la subida a internet de imágenes humillantes de compañeros del colegio; e) la transmisión en línea de secretos de otras personas, información comprometida e imágenes; y e) el grabar por celular videos mientras un grupo se ríe y obliga a otra persona a hacer algo humillante o ridículo (ver tabla 1).

Cabe mencionar además que estas conductas también son las que tienden a manifestarse con mayor intensidad, aunque con menor frecuencia de práctica.

Tabla 1

Prevalencia en la práctica de conductas perpetradoras de ciberbullying

\begin{tabular}{|c|c|c|c|c|c|}
\hline N. & Cuestionario & Nunca & $\begin{array}{c}102 \\
\text { veces }\end{array}$ & $\begin{array}{l}304 \\
\text { veces }\end{array}$ & $\begin{array}{c}5 \text { o más } \\
\text { veces }\end{array}$ \\
\hline 1 & He enviado mensajes amenazantes o insultantes por medio de correo electrónico & $83,3 \%$ & $12 \%$ & $2,8 \%$ & $1,4 \%$ \\
\hline 2 & He enviado mensajes amenazantes o insultantes & $82,4 \%$ & $13,2 \%$ & $2,3 \%$ & $2,1 \%$ \\
\hline 3 & He excluido a alguien de un grupo en línea & $70,1 \%$ & $22,9 \%$ & $4,2 \%$ & $2,8 \%$ \\
\hline 4 & He subido imágenes humillantes de un compañero de clase en internet & $85,2 \%$ & $11,1 \%$ & $2,1 \%$ & $1,6 \%$ \\
\hline 5 & He enviado enlaces electrónicos de imágenes humillantes a otras personas & $89,4 \%$ & $8,6 \%$ & $1,4 \%$ & $0,7 \%$ \\
\hline 6 & $\begin{array}{l}\text { He obtenido la contraseña y enviado correos electrónicos hacia otros en nombre de } \\
\text { esa persona, lo cual puede hacer que esa persona quede mal o cause problemas }\end{array}$ & $89,6 \%$ & $9,3 \%$ & $0,9 \%$ & $0,2 \%$ \\
\hline 7 & $\begin{array}{l}\text { He grabado un video o tomado fotos por celular mientras un grupo se ríe y obliga a } \\
\text { la otra persona a hacer algo humillante o ridículo }\end{array}$ & $87,7 \%$ & $10 \%$ & $1,6 \%$ & $0,7 \%$ \\
\hline 8 & He enviado dichos videos a otra persona & $88,9 \%$ & $9 \%$ & $1,6 \%$ & $0,5 \%$ \\
\hline 9 & $\begin{array}{l}\text { He grabado un video o tomado fotos por celular mientras alguien golpea o lastima } \\
\text { a otra persona }\end{array}$ & $92,1 \%$ & $5,8 \%$ & $0,9 \%$ & $1,2 \%$ \\
\hline 10 & He enviado dichas imágenes grabadas a otras personas & $91 \%$ & $6,3 \%$ & $1,9 \%$ & $0,9 \%$ \\
\hline 11 & $\begin{array}{l}\text { He enviado mensajes que incluyan amenazas o que son muy intimidantes repetiti- } \\
\text { vamente }\end{array}$ & $93,3 \%$ & $5,3 \%$ & $0,7 \%$ & $0,7 \%$ \\
\hline 12 & $\begin{array}{l}\text { He grabado un video o tomado fotos por celular de algún compañero de clase } \\
\text { mientras él o ella está llevando a cabo un comportamiento de connotación sexual }\end{array}$ & $95,1 \%$ & $4,4 \%$ & $0,2 \%$ & $0,2 \%$ \\
\hline 13 & He enviado dichas imágenes a otras personas & $91,9 \%$ & $7,2 \%$ & $0,7 \%$ & $0,2 \%$ \\
\hline 14 & $\begin{array}{l}\text { He transmitido en línea los secretos de otras personas, información comprometida } \\
\text { o imágenes }\end{array}$ & $87,3 \%$ & $10,9 \%$ & $0,9 \%$ & $0,9 \%$ \\
\hline
\end{tabular}




\section{Análisis sobre indicadores de victimización por ciberbullying}

En lo que respecta a los indicadores de victimización como se observa en la tabla 2 , tras la perpetración del acoso virtual se encontró que son víctimas (igual o mayor al 10\% de frecuencia) de situaciones como: a) recibir mensajes amenazantes o humillantes; $b$ ) robo de la identidad de la víctima en el espacio virtual; c) conocer acerca de comentarios de la víctima misma en internet; y d) la difusión de información confidencial de la víctima en internet.

De la misma manera, estos indicadores de victimización se sostienen como situaciones perpetradas contra las víctimas conforme aumenta la intensidad en la agresión.

\section{Distribución de categorías de victimización y perpetración}

De manera general, en cuanto a la presencia de perpetración virtual, es notorio (como se refleja en el gráfico 1) que las conductas perpetradoras leves se encuentran insertas en la práctica común de los participantes, superando el 50\% de los casos analizados, mientras que la presencia moderada es baja y la presencia alta es inexistente. Es decir, existe una práctica de conductas de agredir a otros a través de las TIC de manera rutinaria, aunque no queda claro la intencionalidad ni la consciencia detrás de dichas conductas.
Por otra parte, en la revisión categórica sobre los indicadores de violencia a los que fueron expuestos los participantes se observa que en una mayoría relativa (superior al 50\%) los participantes no se encuentran expuestos a actos lesivos contra su integridad por medio del internet. Sin embargo, el reporte de prácticas perpetradoras contra ellos de forma leve es existente, aunque limitado, mientras que el nivel moderado es escaso y el nivel alto es inexistente.

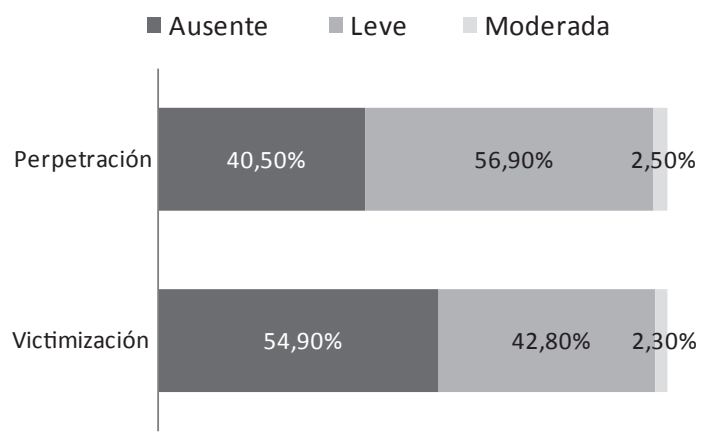

Nota: $X^{2}=125,5 ; p<0,001 ; T-b=0,42 ; p<0,001$

Gráfico 1. Representación porcentual de los niveles de perpetración y de victimización entre los participantes. Elaboración propia

Un aspecto que se debe tomar en cuenta es la estrecha asociación $\left(X^{2}=125,5 ; p<0,001\right)$ entre las conductas perpetradoras ejecutadas por los participantes y las conductas a las que son sometidos. Este

Tabla 2

Prevalencia crítica en los indicadores de victimización tras perpetración

\begin{tabular}{|c|c|c|c|c|c|}
\hline N. ${ }^{\circ}$ & Cuestionario & Nunca & $\begin{array}{c}\text { Entre } 1 \text { o } 2 \\
\text { veces }\end{array}$ & $\begin{array}{c}\text { Entre } 3 \text { o } 4 \\
\text { veces }\end{array}$ & $\begin{array}{c}5 \text { o más } \\
\text { veces }\end{array}$ \\
\hline 1 & Han difundido en internet información confidencial mía & $89,6 \%$ & $8,1 \%$ & $2,3 \%$ & - \\
\hline 2 & Me han grabado realizando conductas sexuales & $97,5 \%$ & $2,1 \%$ & - & $0,5 \%$ \\
\hline 3 & $\begin{array}{l}\text { Me han grabado un video o me han fotografiado mientras me } \\
\text { obligaban a hacer algo humillante }\end{array}$ & $94 \%$ & $5,1 \%$ & $0,5 \%$ & $0,5 \%$ \\
\hline 4 & $\begin{array}{l}\text { Me han grabado un video o me han fotografiado mientras me } \\
\text { agredían físicamente }\end{array}$ & $94,9 \%$ & $3,9 \%$ & $0,5 \%$ & $0,7 \%$ \\
\hline 5 & He recibido mensajes amenazantes o insultantes & $70,1 \%$ & $22,9 \%$ & $4,9 \%$ & $2,1 \%$ \\
\hline 6 & Han colgado imágenes mías humillantes en internet & $91,7 \%$ & $6,5 \%$ & $1,4 \%$ & 0,5 \\
\hline 7 & Han colgado comentarios sobre mí en internet & $83,1 \%$ & $12,5 \%$ & $2,3 \%$ & $2,1 \%$ \\
\hline 8 & Han usado mi identidad & $81,5 \%$ & $14,4 \%$ & $2,5 \%$ & $1,6 \%$ \\
\hline 9 & He recibido masivamente mensajes muy amenazantes & $88,7 \%$ & $7,6 \%$ & ,9\% & $2,8 \%$ \\
\hline
\end{tabular}


dato, junto con el análisis de correlación, refleja que existe una relación moderada en esta dinámica y establece la tendencia entre los participantes en ser agresor o perpetrador de ciberbullying y también ser víctima de las mismas prácticas.

\section{Análisis comparativo en la práctica y victimización del ciberbullying}

\section{Análisis comparativo por sexo}

Como se puede observar en la tabla 3 , en el análisis de perpetración se muestra tanto en hombres como en mujeres una presencia baja de conductas perpetradoras de forma virtual. En esta línea de análisis, los hombres muestran una mayor manifestación de perpetración que las mujeres, aunque estadísticamente no muestren diferencias significativas $(p>0,5)$. Es decir, en este aspecto del análisis los hombres y las mujeres agreden virtualmente de la misma forma y con la misma intensidad.

Una situación similar se puede observar en el análisis de la presencia e intensidad de indicadores de violencia virtual. Aunque en este caso, las mujeres reporten ligeramente mayor perpetración que los hombres, pero sin mostrar diferencias estadísticamente significativas $(p>0,5)$. De tal manera, se puede estimar que la victimización por ciberbullying es similar en la práctica e intensidad.

\section{Análisis comparativo por tipo de institución educativa}

Si se establece un análisis comparado por el tipo de financiación de las instituciones educativas (públicas o privadas), como lo refleja la tabla 4 , se puede considerar la existencia de diferencias $(p<0,05)$ en la perpetración, pues se observa que la práctica es más marcada en los participantes procedentes de colegios privados que públicos con un tamaño del efecto pequeño.

Mientras tanto, a nivel de indicadores de victimización los puntajes entre participantes de colegios públicos y privados no muestran diferencias significativas, aunque la victimización es más notoria en escuelas privadas que públicas. De alguna manera, esto puede ser explicado precisamente por la tendencia a una mayor práctica de perpetración en los mismos centros privados.

\section{Discusión}

Los objetivos del estudio fueron conocer las prácticas más recurrentes de ciberbullying, la prevalencia de conductas perpetradoras y de victimización cibernética, así como las diferencias por sexo y tipo de escuela en los actores de ciberbullying.

Sobre las conductas perpetradoras encontradas en el estudio, las de mayor prevalencia son: a) la

Tabla 3

Análisis comparativo por sexo de las puntuaciones de ciberbullying

\begin{tabular}{|c|c|c|c|c|c|c|}
\hline \multirow{2}{*}{ Variables } & \multirow{2}{*}{$\begin{array}{c}\text { Hombres } \\
\text { Media (DT) }\end{array}$} & \multirow{2}{*}{$\begin{array}{c}\text { Mujeres } \\
\text { Media (DT) }\end{array}$} & \multirow{2}{*}{ F } & \multicolumn{2}{|c|}{ Contraste } & \multirow{2}{*}{$\mathbf{g}_{\text {(ajust.) }}$} \\
\hline & & & & Dif. & $\mathbf{t}_{(419)} ; \mathbf{p}$ & \\
\hline Perpetración & $2,48(3,72)$ & $2,05(3,93)$ & 1,35 & 0,42 & $-0,18 ; 0,26$ & 0,11 \\
\hline Victimización & $1,43(2,53)$ & $1,48(2,68)$ & 0,49 & $-0,47$ & 1,$12 ; 0,86$ & 0,02 \\
\hline
\end{tabular}

Nota. Elaboración propia

Tabla 4

Análisis comparativo por tipo de institución educativa de las puntuaciones de ciberbullying

\begin{tabular}{|c|c|c|c|c|c|c|}
\hline \multirow{2}{*}{ Variables } & \multirow{2}{*}{$\begin{array}{c}\text { Públicas } \\
\text { Media (DT) }\end{array}$} & \multirow{2}{*}{$\frac{\text { Privadas }}{\text { Media (DT) }}$} & \multirow{2}{*}{$\mathrm{F}$} & \multicolumn{2}{|c|}{ Contraste } & \multirow{2}{*}{$\mathbf{g}_{\text {(ajust. }}$} \\
\hline & & & & Dif. & $t_{(419)} ; p$ & \\
\hline Perpetración & $1,97(3,30)$ & $3,25(5,28)$ & $13,05^{\star * *}$ & $-1,28$ & $-2,18 ; 0,03$ & 0,34 \\
\hline Victimización & $1,38(2,62)$ & $1,74(2,53)$ & 0,49 & $-0,35$ & $-1,14 ; 0,26$ & 0,14 \\
\hline
\end{tabular}

Nota. ${ }^{* * *} p<0,001$. Elaboración propia 
exclusión de alguien de un grupo en línea y el envío de mensajes amenazantes e insultantes por medio del correo electrónico; b) la transmisión en línea de secretos de otras personas, información comprometida e imágenes; y c) haber grabado por celular videos mientras un grupo se ríe y obliga a otra persona a hacer algo humillante o ridículo. Hallazgos similares en este estudio se encuentran en los trabajos previos de Rivers y Noret (2010), Singhal y Bansal (2013), Livingstone y Smith (2014) y De Tejada, Muñoz y Rus (2018). Se infiere un patrón comportamental de los perpetradores de tipo intimidatorio aunque no expresamente violento en términos físicos.

La práctica de al menos una conducta de ciberbullying por el $56,9 \%$ de los participantes evidencia un comportamiento preocupante en esta muestra analizada si se compara con los datos de Tokunaga (2010) o con reportes en Chile (Varela et al., 2018), Taiwán (Chang et al. 2013) o México y otros países (Gámex-Gaudi, Villa-George y Calvete, 2014). Es probable que la incorporación de las tecnologías ayude a expandir los escenarios donde se pueden establecer comportamientos de violencia debido a la facilidad para transgedir las normas sociales de convivencia (Donegan, 2012).

Por otra parte, las víctimas del ciberbullying de manera más generalizada se ven sometidas a la recepción de mensajes amenazantes o humillantes, al robo de su identidad en el espacio virtual, al conocimiento o descubrimiento de comentarios en internet y a la difusión de su información confidencial y personal en línea. Los estudios referenciales de Ybarra y Mitchell (2004), Menesini, Nocentini y Calussi (2011) y Livingstone y Smith (2014) arrojan situaciones similares que reportaron las víctimas en otros contextos. También, se aprecia que la práctica de la perpetración y las reacciones de victimización se relacionan con la humillación y el envío de información, aunque no con contenido sexual expreso o con la manifestación marcada de violencia física o amenaza de la práctica de la violencia física contra la víctima.

La incidencia de indicadores de victimización leve tras el ciberbullying está extendida ampliamente entre los participantes, aunque de carácter relativamente leve, lo que hace difícil tomar estos elementos como significativos para considerarlos un problema generalizado que pueda despertar las alarmas sociales, dada su intensidad y frecuencia. Este bordea el $42,8 \%$ aproximadamente; aun así, estos concuerdan con estudios similares (Kowalski et al., 2014 y Eurostat, 2015). Debe también estimarse que datos en el contexto ecuatoriano son superiores a los encontrados previamente por Mosquera et al. (2016), principalmente porque se hizo el estudio en un solo centro educativo.

Se debe reconocer un leve porcentaje de víctimas moderadas de acoso $(2,3 \%)$ con un nivel inferior a lo observado en los trabajos de Sevilla (2011) y Del Río et al. (2009). En estos casos, es relevante considerar las consecuencias del acoso a las víctimas, ya que tienen una correspodencia con problemas de salud mental (Chang et al., 2013; Kessel et al., 2012; Cetin et al., 2012) y de escolaridad (Yousef y Bellamy, 2015). Cabe señalar la distancia que existe entre los individuos acosadores y las víctimas de ciberbullying, ya que la práctica de acoso es más prevalente en el individuo que la recepción de ataques imtimidatorios o acosadores. Los agresores son más agresores que víctimas y viceversa.

No se encontraron diferencias por género, ni en las conductas de perpetración ni en los indicadores de victimización de ciberbullying. Previamente, los trabajos de Qing (2006) y Gámex-Gaudi, Villa-George y Calvete (2014) establecian la presencia de diferencias por género: los hombres mostraban mayor comportamiento agresor y las mujeres, mayores niveles de acoso; sin embargo, para el presente caso, esto no sucedió así. Es probable que esto sea explicado por los cambios sociales que se dan por el género y la práctica generalizada del acoso virtual.

Sí se hallaron diferencias entre los estudiantes que proceden de instituciones educativas privadas y públicas, presentándose mayor intensidad de la perpetración entre los procedentes de instituciones privadas. No se han encontrado estudios referenciales que puedan dar una perspectiva de este particular, aunque el fenómeno podría ser explicado por las condiciones socioeconómicas de las familias, la cantidad de equipo informático presente y las características ambientales y sociales de las instituciones que han sido encontrados en situaciones de riesgo, consumo de drogas y prácticas sexuales riesgosas (Moreta et al., 2017).

Los resultados del presente estudio, además, tienen implicación de la situación del acoso virtual en los entornos educativos del Ecuador, también acerca de la práctica y la asimilación de la violencia real o virtual en los contextos socioeducativos por parte de los adolescentes, y en especial en el entendimiento cultural de la violencia y el acoso escolar y en otras formas de agresión. Pero también las diferencias que pueden existir en los entornos educativos de carácter 
público y privado. Así mismo, estos datos ayudan a presenciar un fenómeno generalizado crónico, que por el grado de manejo de la violencia en el imaginario colectivo no logra despertar adecuadamente las alarmas sociales que desalienten y mitiguen los efectos de la práctica del acoso.

A partir de estos resultados, se pueden explorar los efectos psicológicos y sociales de la práctica de la violencia y la perpetración real y virtual en los centros educativos, los mecanismos de afrontamiento de las víctimas al ciberbullying y las justificaciones de los perpetradores para su cometimiento, así como las respuestas socioeducativas que se pueden implementar para su contención.

\section{Limitaciones y recomendaciones a futuro}

Entre las limitaciones del presente estudio se debe comentar que no se realizó un análisis sobre el acceso, el manejo y la supervisión que tienen los adolescentes hacia las TIC. De la misma manera, no se contempló el manejo, el número y el dominio de las redes sociales que tienen los participantes, por lo que a futuro se recomienda indagar sobre estos elementos.

Con respecto a la fiabilidad de la prueba, no se tomaron en consideración otros criterios de fiabilidad como la estabilidad temporal de la prueba, la validez de convergencia con otros instrumentos y la validez discriminante de la prueba. Aunque los resultados dan muestra de una buena fiabilidad de la prueba y que es concordante con otros estudios, estos requieren ser tomados con cautela y realizar otros estudios de verificación de fiabilidad de la prueba.

El estudio no consideró los aspectos de medición del ciberbullying con otras variables relacionadas con el entorno socioeducativo, como el clima escolar y familiar, el rendimiento académico y la motivación escolar, entre otros. $\mathrm{O}$ variables de aspecto individual como los estados emocionales, el control de impulsos y los motivos para acosar, entre otros. Razón por la cual a futuro se sugiere complementar estos datos con las variables sugeridas.

\section{Referencias}

Bauman, S., Cross, D. y Walker, J. L. (2013). Principles of cyberbullying research: Definitions, measures and methodology. Nueva York: Routledge.
Calvete, E., Orue, I., Estévez, A., Villardón, L. y Padilla, P. (2010). Cyberbullying in adolescents: Modalities and aggressors' profile. Computers in Human Behavior, 26, 1128-1135. doi: https://doi.org/10.1016/j.chb.2010. 03.017

Castells, M. (2010). El impacto de internet en la sociedad: una perspectiva global. Los Ángeles: University of Southern California.

Castillo-Pulido, L. E. (2011). El acoso escolar. De las causas, origen y manifestaciones a la pregunta por el sentido que le otorgan los actores. Revista Internacional de Investigación en Educación, 4(8), 415-428.

Cetin, B., Eroglu, Y., Peker, A., Akbaba, S. y Pepsoy, S. (2012). The investigation of relationship among relational-interdependent self-construal, cyberbullying, and psychological disharmony in adolescents: An investigation of structural equation modelling. Educational Sciences, 12(2), 646-653.

Chang, F. C., Lee, C. M., Chiu, C. H., Hsi, W. Y., Huang, T. F., y Pan, Y. C. (2013). Relationships among cyberbullying, school bullying, and mental health in Taiwanese adolescents. Journal of School Health, 83(6), 454-462. doi: http://doi.org/10.1111/josh.12050

Cohen, J. (1994). The Earth is round ( $\mathrm{p}<.05)$. American Psychologist, 49(12), 997-1003.

De Tejada, J. D., Muñoz, M. Á. y Rus, T. I. (2018). Ciberbullying: análisis comparativo entre menores de España y Francia. Revista de Humanidades, 33(1), 173-188. doi: https://doi.org/10.5944/rdh.33.2018

Del Río Pérez, J., Bringue Sala, X., Sádaba Chalezquer, C. y González González, D. (2009). Cyberbullying: un análisis comparativo en estudiantes de Argetina, Brasil, Chile, Colombia, México, Perú y Venezuela. V Congrés Internacional Comunicació i Realitat (pp. 307-316). Navarra: Departamento de Comunicación Audiovisual y Publicidad y Literatura, Foro Generaciones Interactivas, Universidad de Navarra.

Di Napoli, P. (2016). La violencia en las escuelas secundarias desde tres perspectivas de análisis. Hacia un estado del arte. Revista del Instituto de Estudios en Educación Universidad del Norte, 1(24), 61-84. doi: http://doi. org/10.14482/zp.24.8722

Donegan, R. (2012). Bullying and cyberbullying: History, statistics, law, prevention and analysis. The Elon Journal of Undergraduate Research in Communications, $3(1), 33-42$.

Estévez, A., Villardón, L., Calvete, E., Padilla, P., y Orue, I. (2010). Adolescentes víctimas de cyberbullying: preva- 
lencia y características. Behavioral Psychology/Psicología Conductual, 18(1), 73-89.

Eurostat. (2015). Acerca de nosotros: Eurostat. Recuperado de http://ec.europa.eu/eurostat/documents/3217494/ 7018888/KS-HA-15-001-EN-N.pdf/6f0d4095-5e7a4aab-af28-d255e2bcb395

Gámex-Gaudi, M., Villa-George, F. y Calvete, E. (2014). Psychometric properties of the Cyberbullying Questionnaire (свQ) among Mexican adolescents. Violence and Victims, 29(2), 233-247. doi: http://doi.org/ 10.1891/0886-6708.VV-D-12-00163R1

Gámez-Guadix, M., Gini, G. y Calvete, E. (2015). Stability of cyberbullying victimization among adolescents: Prevalence and association with bully-victim status and psychosocial adjustment. Computers in human behavior, 53, 140-148. doi: https://doi.org/10.1016/j. chb.2015.07.007

Garaigordobil, M. (2015). Ciberbullying en adolescentes y jóvenes del País Vasco: cambios con la edad. Anales de psicología, 31(3), 1069-1076. doi: http://dx.doi. org/10.6018/analesps.31.3.179151

IвM Corporation (2012). IBM SPSS Statistics for Windows. Armonk: IBM Corporation.

Kessel, S., O’Donnell, L., Stueve, A. y Coulter, R. W. (2012). Cyberbullying, school bullying, and psychological distress: A regional census of high school students. American Journal of Public Health, 102(1), 171-177. doi: http://doi.org/10.2105/AJPH.2011.300308

Kowalski, R., Giumetti, G., Schoeder, A. y Lattanner, M. (2014). Bullying in the digital age: A critical review and meta-analysis of cyberbullying research among youth. Psychological Bulletin, 140(4), 1073-1137. doi: http:// doi.org/10.1037/a0035618.

Livingstone, S. y Smith, P. K. (2014). Annual Research Review. Harms experienced by child users of online and mobile technologies: the nature, prevalence and management of sexual and aggressive risks in the digital age. Journal of Child Psychology and Psychiatry, 55(6), 635-654. doi: http://doi.org/10.1111/jcpp.12197

Menesini, E., Nocentini, A. y Calussi, P. (2011). The measurement of cyberbullying: Dimensional structure and relative item severity and discrimination. Cyberpsychology, Behavior and Social Networking, 14(5), 267274. doi: http://doi.org/10.1089/cyber.2010.0002

Montero, I. y León, O. (2007). A guide for naming research studies in Psychology. International Journal of Clinical and Health Psychology, 7(3), 847-862.

Moreta, R., Reyes, C., Mayorga, M. y León-Tamayo, L. (2017). Estimación sobre niveles y factores de riesgo psicosocial en adolescentes escolarizados de Ambato, Ecuador. Pensando Psicología, 8(2), 29-40. doi: https:// doi.org/10.16925/pe.v13i22.1986

Mosquera, J. E., Moscoso, G. A., Samaniego, M. O. y Vallejo, L. E. (2016). Características del ciberbullying en los estudiantes de la unidad educativa César Dávila Andrade. Cuenca, Ecuador, 2013. Revista de la Facultad de Ciencias Médicas, 33(3), 41-49.

Olweus, D. (2016). Cyber bullying. A critical overview. En: B. Bushman (ed.), Aggression and violence: A social psychological perspective (pp. 225-239). Nueva York: A Psychology Press Book.

Pardo, A. y San Martín, R. (2010). Análisis de datos en Ciencias Sociales y de la Salud II. Madrid: Síntesis.

Pierskalla, J. H. y Hollenbach, F. M. (2013). Technology and collective action: The effect of cell phone coverage on political violence in Africa. American Political Science Review, 107(2), 207-224. doi: http://doi.org/10.1017/ S0003055413000075

Qing,L.(2006).Cyberbullyinginschools. A research ofgender differences. School Psychology International, 27(2), 157 170. doi: https://doi.org/10.1177/0143034306064547

Rivers, I. y Noret, N. (2010). 'I h8 u': Findings from a fiveyear study of text and email bullying. British Educational Research Journal, 36(4), 643-671. doi: 10.1080/ 01411920903071918

Rodríguez-Correa, M. y Arroyo, M. (2014). Las TIC al servicio de la inclusión educativa. Digital Education Review, 25, 108-126.

Roig, A. E., López, M. y Álvarez, G. (2017). Una mirada crítica sobre los nativos digitales: análisis de los usos formales de TIC entre estudiantes universitarios. Revista Q, 9(17), 1-19. Disponible en: https://goo.gl/39S1n6

Sevilla, A. (2011, noviembre 19). De 9.000 niños y adolescentes mendocinos, 1.800 fueron agredidos por Internet o SMS. Diario UNO. Recuperado el 2 de enero de 2017, de http://www.diariouno.com.ar/pais/de-9000ninos-y-adolescentes-mendocinos-1800-fueron-agredidos-internet-o-sms-20111119-n82606.html

Shu-Ling, L., Renmin, Y. y Kuo-Pao, C. (2008). Bullying in middle schools: An Asian-Pacific regional study. Asia Pacific Education Review, 9(4), 393-405. doi: http://doi. org/10.1007/BF03025666

Simone, P., Smith, P. K. y Blumberg, H. H. (2012). Investigating legal aspects of cyberbullying. Psicothema, 24(4), 640-645.

Singhal, P. y Bansal, A. (2013). Improved textual cyberbullying detection using data mining. International Jour- 
nal of Information and Computation Technology, 3(6), 569-575.

Tokunaga, R. S. (2010). Following you home from school: A critical review and synthesis of research on cyberbullying victimization. Computers in Human Behavior, 26, 277-287. doi: https://doi.org/10.1016/j.chb. 2009.11.014

Varela, J., Guzmán Piña, J., Alfaro, J. y Reyes, F. (2018). Bullying, cyberbullying, student life satisfaction and the community of Chilean adolescents. Applied Research in Quality of Life, 1-16. doi: https://doi.org/10.1007/ s11482-018-9610-7
Ybarra, M. L. y Mitchell, K. J. (2004). Online aggressor/targets, aggressors, and targets: A comparison of associated youth characteristics. Journal of Child Psychology and Psychiatry, and Allied Disciplines, 45(7), 1308-1316. doi: http://doi.org/10.1111/j.1469-7610.2004.00328.x

Yousef, W. S. y Bellamy, A. (2015). El impacto del ciberbullying sobre la autoestima y el rendimiento académico de estudiantes árabe-americanos de secundaria. Electronic Journal of Research in Educational Psychology, 13(37), 463-482. doi: http://dx.doi.org/10.14204/ejrep. $43 / 17075$ 